\title{
Sistema de ClassificaÇão Fuzzy PaRA O Risco de InfestaÇÃo POR Plantas Daninhas Considerando a sua Variabilidade Espacial ${ }^{1}$
}

\author{
Fuzzy Classification System for Risk of Weed Infestation Considering Spatial Variability
}

BRESSAN, G.M. ${ }^{2}$, KOENIGKAN, L.V. ${ }^{2}$, OLIVEIRA, V.A. ${ }^{3}$, CRUVINEL, P.E. ${ }^{4}$ e KARAM, D. ${ }^{5}$

\begin{abstract}
RESUMO - Este artigo trata do problema de classificação do risco de infestação por plantas daninhas usando técnicas geoestatísticas, análise de imagens e modelos de classificação fuzzy. Os principais atributos utilizados para descrever a infestação incluem a densidade de sementes, bem como a sua extensão, a cobertura foliar e a agressividade das plantas daninhas em cada região. A densidade de sementes reflete a produção de sementes por unidade de área, e a sua extensão, a influência das sementes vizinhas; a cobertura foliar indica a extensão dos agrupamentos das plantas daninhas emergentes; e a agressividade descreve a porcentagem de ocupação de espécies com alta capacidade de produção de sementes. Os dados da densidade de sementes, da cobertura foliar e da agressividade para as diferentes regiões são obtidos a partir de simulação com modelos matemáticos de populações. Neste artigo propõe-se um sistema de classificação fuzzy utilizando os atributos descritos para inferir os riscos de infestação de regiões da cultura por plantas daninhas. Resultados de simulação são apresentados para ilustrar o uso desse sistema na aplicação localizada de herbicida.
\end{abstract}

Palavras-chave: lógica fuzzy, geoestatística, dinâmica populacional, mapas, análise de imagem.

\begin{abstract}
This paper deals with the problem of classifying the risk of infestation by weeds in a field using geoestatistics techniques, image analysis and fuzzy classification models. The main attributes used to describe the infestation include seed density, seed density patch, weed cover and aggressivity to produce seeds in each region. Seed density reflects seed production per area unit; seed density patch reflects the influence of the neighbouring seeds in a clustering; weed cover indicates the extension of the emergent weed plant clusterings; and, finally, aggressivity describes the percentage of occupation of species with high weed seed production capacity. Data for seed density, weed cover and aggressivity for the different regions are obtained from mathematical models. In this paper, a fuzzy classification system using the attributes described is proposed to infer about the infestation risks of crop regions by weed plants. Simulation results of the proposed risk classification system are presented to illustrate its use in the site-specific herbicide application.
\end{abstract}

Keywords: fuzzy logic, geoestatistics, populational dynamics, maps, image processing.

\section{INTRODUÇÃO}

Procedimentos agrícolas podem modificar o equilíbrio ecológico, devido à maneira como a terra é lavrada, tornando propícia a explosão populacional de certas plantas, chamadas plantas daninhas (Shiratsuchi \& Christoffoleti,
2001). A presença dessas plantas pode interferir na operacionalização do sistema de produção, causando prejuízos na produtividade (Baio \& Balastreire, 2001). A infestação por plantas daninhas normalmente não ocorre de modo uniforme nas áreas agrícolas, sendo possível o mapeamento de sua variabilidade espacial

Recebido para publicação em 15.5.2005 e na forma revisada em 5.5.2006.

2 Aluno de pós-graduação em Engenharia Elétrica, EESC/USP; ${ }^{3}$ Profa. do Dep. de Engenharia Elétrica - EESC/USP, Av. Trabalhador São-carlense, 400, 13566-590 São Carlos-SP. ${ }^{4}$ EMBRAPA - Instrumentação Agropecuária, $15025-400$ São Carlos-SP. ${ }^{5}$ EMBRAPA - Milho e Sorgo, 35701-970 Sete Lagoas-MG. 
com a utilização de ferramentas de agricultura de precisão, permitindo a tomada de decisão sobre o melhor manejo para cada local do campo (Cruvinel et al., 2004; Baio \& Balastreire, 2001). A previsão da dispersão dessas plantas pode ser eficientemente utilizada na prevenção de infestações com a aplicação localizada de herbicidas, para reduzir o custo de aplicação de insumos e, por conseqüência, o impacto ambiental (Aitkenhead et al., 2003).

Considerando os altos custos, a subjetividade e a inconsistência associados com a classificação manual de produtos, as indústrias têm sido levadas a automatizar o processo de classificação. Como a maioria das técnicas de tomadas de decisão avalia o risco por valores lingüísticos, a lógica fuzzy fornece uma alternativa para tratar com as incertezas associadas à população das plantas. Em particular, as fronteiras entre classes não são claramente definidas, e os sistemas de classificação fuzzy podem tratar dessa questão (Chiang \& Hsu, 2002). Esses sistemas são aplicados em problemas de classificação em que vetores de entrada são combinados por regras e atribuídos a um dos conjuntos de classes de saída especificado.

Em Kavdir \& Guyer (2003), a lógica fuzzy foi aplicada como suporte à tomada de decisão para classificar maçãs com respeito à sua qualidade. Atributos de qualidade como cor, tamanho e manchas da maçã foram medidos por diferentes equipamentos. O mesmo conjunto de maçãs foi classificado por um especialista e por um sistema fuzzy. Os resultados obtidos pelo sistema fuzzy mostraram $89 \%$ de concordância com os resultados do especialista.

Em Peixoto et al. (2004) é elaborado um modelo do tipo presa-predador que descreve a interação de pulgões (presas) e joaninhas (predador) na citricultura, em que os pulgões são considerados agentes transmissores da morte súbita de citros (doença causada por vírus). Utiliza-se nesse trabalho um sistema fuzzy baseado em regras lingüísticas para estabelecer a relação entre as variáveis de entrada, que são o espaço percorrido pelo pulgão e a intensidade do vento. Considerando a quantidade média de pulgões e joaninhas em um ramo de laranjeira, simulações foram feitas observando-se a variação da quantidade de presas e do potencial de predação, que são as variáveis de saída do sistema fuzzy.

Em Yang et al. (2003) utiliza-se a lógica fuzzy para associar dados de cobertura foliar, obtidos por imagens fotografadas em campo, a três níveis de aplicação localizada de herbicida.

Neste artigo, um sistema de classificação fuzzy para inferir o risco de infestação por plantas daninhas, usando krigagem e análise de imagens, é proposto. Resultados de simulação para a classificação do risco levando em conta a variabilidade da população de plantas em regiões são apresentados e comparados com aqueles obtidos para toda a área, considerando um único risco de infestação.

Este trabalho é organizado como segue. Na seção Material e Métodos, os modelos dinâmicos usados na simulação de dados para a população de plantas daninhas, o método de obtenção dos mapas de densidade e cobertura foliar e a estrutura do sistema de classificação fuzzy proposto são resumidamente descritos. Na seção Resultados e Discussões são detalhados os procedimentos empregados para obtenção dos riscos de infestação a partir de dados de populações simulados.

\section{MATERIAL E MÉTODOS}

\section{Modelos dinâmicos de sementes}

A geração de plantas via sementes requer que uma porção das sementes contidas no solo seja fisiologicamente capaz de germinar em condições ambientais apropriadas. O comportamento da dinâmica populacional depende especialmente da taxa de germinação da semente. A densidade de sementes, dada pelo número de sementes por área nos sucessivos anos, pode ser obtida recursivamente a partir do número de sementes do ano inicial observado (Gonzalez-Andujar, 1996):

$$
X_{t}=(1-c) \operatorname{sg} X_{t-1}\left(1+a g X_{t-1}\right)^{-b} \quad \text { (eq. 1) }
$$

em que $X_{t}=$ densidade de sementes (sementes/ $\left.\mathrm{m}^{2}\right) ; t=$ geração da planta; $g=$ taxa de germinação das sementes; $s=$ número de sementes por planta em ausência de competição; $a=$ taxa de declínio da população de sementes com o 
aumento da densidade de plantas; $b=$ taxa que reflete a intensidade do efeito da densidade de plantas na produção de sementes; e $c=$ taxa de controle da população, a qual permite a redução na sobrevivência das sementes.

A partir de medidas de controle com o uso de herbicidas e dentro da margem de extinção da população de acordo com a taxa de germinação $(g)$, a extinção pode ser obtida somente com $100 \%$ de controle, ou seja, $c=1$ na equação 1 . Contudo, o controle médio alcançado nas condições do campo em geral não ultrapassa 90\%, dada a situação paradoxal de que não se pode erradicar a população apesar do alto nível de controle alcançado (Gonzalez-Andujar, 1996). O comportamento da densidade de sementes de plantas daninhas em função das gerações sucessivas da planta, dada pela equação 1 , com taxa de germinação $g=0,1$, pode ser observado na Figura 1, onde se pode notar a estabilização da densidade em níveis baixos, por efeito da taxa de controle $(c)$.

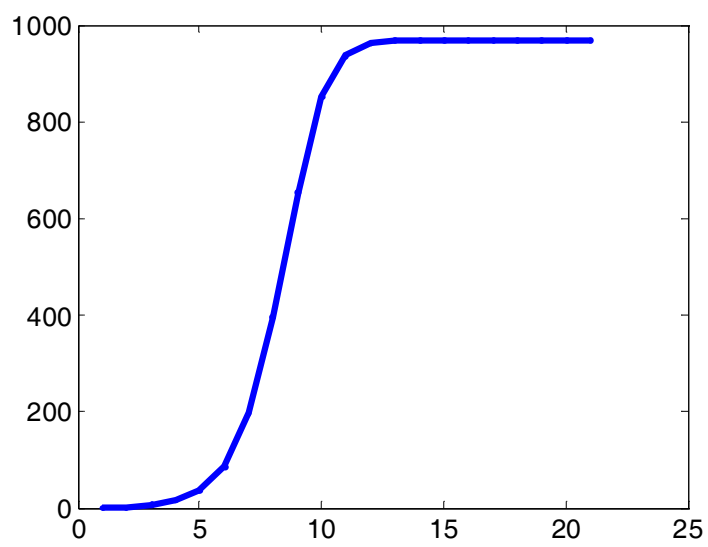

Figura 1 - Densidade de sementes em função das gerações sucessivas da planta para $g=0,1, s=248, a=4,0 \times 10^{-3}, b=$ 2,77 e $c=0,9$.

A cobertura foliar é definida como a proporção de área do solo ocupada pela projeção vertical da parte área de uma espécie (Rizzardi \& Fleck, 2004). A densidade de plantas na geração $t-1$, denotada por $Y_{t-1}$, pode ser obtida a partir das sementes que germinaram em $t-1$ :

$$
Y_{t-1}=g X_{t-1}
$$

Para obter mapas de densidades, o emprego de técnicas geoestatísticas se faz necessário, com os objetivos de avaliar a dependência espacial dos dados e estimar valores para locais não-observados.

\section{Estimativas de mapas de densidade}

Os métodos de estatística clássica assumem que a variabilidade das observações é aleatória e espacialmente independente. No entanto, a continuidade espacial existe na maioria das observações de atributos do solo e, conseqüentemente, os valores em locais mais próximos entre si são mais semelhantes, até um determinado limite, que aqueles tomados a maiores distâncias. Portanto, os dados do solo não podem ser tratados como independentes, sendo necessária uma análise estatística que considere essa característica (Vieira et al., 1981).

As técnicas de interpolação têm sido usadas em agricultura de precisão para inferir valores de pontos em locais não-observados. Nesse contexto, objetiva-se utilizar técnicas de estimação a partir de observações obtidas em localização conhecida, com a finalidade de obter mapas de densidade. O método de estimativa básico utilizado é o da krigagem (Isaaks \& Srivastava, 1989), que é uma técnica de interpolação que faz ótima a estimação de variáveis regionalizadas, a partir de uma análise variográfica.

O semivariograma associado a atributos do solo mostra a medida do grau de dependência espacial entre observações; para sua construção são usadas simplesmente as diferenças ao quadrado de pares dos valores obtidos. Suponha que no local $i$, onde cada $i$ representa um par de coordenadas $(x(i), y(i))$, $z(i)$ é o valor obtido para um atributo do solo, e para o local $i+h, z(i+h)$ é o valor obtido para o mesmo atributo. Se houver $N$ pares de observações separadas pela distância $h$, temse, então, a equação do semivariograma:

$$
\gamma^{*}(h)=\frac{1}{2 N_{h}} \sum_{i=1}^{N_{h}}[z(i+h)-z(i)]^{2}, \quad \text { (eq. 3) }
$$

em que $h=$ passo básico; e $N_{h-}=$ número de pares de pontos separados pela distância $h$, o qual deve ser um número grande o suficiente para fornecer um resultado consistente. 
Os valores nas localizações $i=1, \ldots, N_{h}$ gerados pela equação 3 são ajustados a um modelo de semivariograma teórico, o qual é uma curva que proporciona a máxima correlação entre estes valores. Geralmente, o semivariograma é uma função monótona crescente, alcançando um valor-limite, obtido para um valor de $h$ conhecido como alcance. As observações correspondentes a distâncias maiores que esse alcance possuem correlação nula. Dessa forma, a dependência espacial diminui à medida que a distância $h$ aumenta e se anula a uma certa distância, após o alcance.

O estimador de krigagem é linear, porque suas estimativas são combinações lineares ponderadas das observações; é não-enviesado, porque a média residual é próxima a zero e, além disso, minimiza a variância dos erros, o que a difere dos outros métodos de estimação (Isaaks \& Srivastava, 1989). O estimador linear é assim definido por Carvalho \& Vieira (2001):

$$
Z^{*}(u)=\sum_{i=1}^{N} \lambda_{i}(u) Z\left(u_{i}\right)
$$

sujeito a:

$$
\sum_{i=1}^{N} \lambda_{i}(u)=1,
$$

em que os $N$ pesos $\lambda_{i}(u)$ são determinados de tal forma que a variância do erro seja mínima e $Z\left(u_{i}\right)$ são as observações tomadas em uma vizinhança de $Z(u)$ que é usada para a estimação de $Z(u)$. Encontrar a solução do estimador é, portanto, encontrar os pesos $\lambda_{i}(u), i=1, \ldots, N$ a partir do seguinte sistema:

$$
\left[\begin{array}{l}
\lambda_{1} \\
\vdots \\
\lambda_{N} \\
\mu
\end{array}\right]=\left[\begin{array}{llcc}
\gamma_{11} & \cdots & \gamma_{1 N} & 1 \\
\vdots & \cdots & & \vdots \\
\gamma_{N 1} & \cdots & \gamma_{N N} & 1 \\
1 & \cdots & 1 & 0
\end{array}\right]^{-1}\left[\begin{array}{l}
\gamma_{10} \\
\vdots \\
\gamma_{N 0} \\
1
\end{array}\right], \quad \text { (eq. 5) }
$$

com $\gamma_{i j}, i, j=0,1, \ldots, N$ sendo o valor predito pelo modelo teórico de variograma, dado por $\gamma_{i j}=C+$ $C_{0}-\gamma(h)$, expresso por $\gamma(h) ; \gamma_{i 0}$, a posição que se pretende estimar; $C$, a variância espacial; $C_{0}$, o efeito pepita (parâmetros obtidos do variograma); e $h$, a distância entre as observações.

\section{Sistemas de classificação fuzzy e atributos da infestação}

Levando em conta a natureza das infestações agrícolas e tendo em vista os modelos incertos associados à dinâmica da população de plantas, os sistemas de classificação fuzzy são empregados para representar classes. Os problemas de classificação preocupam-se em determinar as classes para os exemplos dados. Cada exemplo pode ser descrito em termos de um conjunto de atributos, que são usados como base para a classificação. Portanto, dado um conjunto arbitrário de dados, a questão mais importante é identificar seus atributos-chave.

Um classificador fuzzy possui quatro componentes: um processador de entrada (ou fuzzificador), um conjunto de regras lingüísticas, um método de inferência fuzzy e um processador de saída (ou defuzzificador), gerando um número real como saída. Inferência fuzzy é o método que interpreta as variáveis de entrada e, baseado em algum conjunto de regras, designa valores para a saída. O processo de inferência fuzzy envolve funções matemáticas que definem o grau de pertinência de um elemento a um conjunto fuzzy, chamadas funções de pertinência, operadores fuzzy (E corresponde ao operador de minimo e OU corresponde ao operador de máximo) e regras SE-ENTÃO, em que a parte SE da regra é chamada de premissa e a parte ENTÃO é chamada de conseqüente (Pedrycz \& Gomide, 1998).

As regras de classificação fuzzy são definidas da seguinte forma:

\section{SE atributo é condição ENTÃO classe}

em que atributo é o vetor $X_{j k} \in\left\{X_{1 k}, \ldots, X_{n k}\right\}$, condição é o conjunto de funções de pertinência $A_{i j} \in\left\{A_{i 1}, \ldots, A_{i n}\right\}$ e classe é $c_{i} \in\left\{c_{1}, \ldots, c_{C}\right\}, \operatorname{com} i=$ $1, \ldots C$ o número de classes, $j=1, \ldots, n$ o número de atributos e $k=1, \ldots, N$ as realizações.

A partir do conhecimento existente de uma determinada região da cultura, são definidos quatro atributos, $n=4$, para as regras de classificação do risco de infestação, explicados a seguir.

Atributo 1: Valor médio da densidade de sementes de plantas daninhas estimado na 
geração t em cada região. Este atributo reflete os focos de maior e menor produção de sementes de plantas daninhas, que cairão sobre o solo, podendo germinar e influenciar a próxima geração.

Atributo 2: Cobertura foliar da planta daninha na geração $t-1$ por região. Este atributo indica a porcentagem de ocupação na região das plantas daninhas emergentes. A cobertura foliar pode ser obtida tomando-se a densidade de sementes na geração $t-1$, multiplicada pela taxa de germinação $g$, como na equação 3.

Atributo 3: Extensão média dos agrupamento de sementes em cada região. Este atributo representa como as sementes contribuem para sua proliferação na vizinhança e reflete o tamanho do agrupamento de um determinado valor de densidade de sementes, de forma que pode ser encontrada alta densidade de sementes, mas com pequeno agrupamento ou mancha.

Atributo 4: Agressividade da planta daninha. Este atributo reflete a alta produção de sementes das espécies de plantas daninhas, como, por exemplo, as espécies de gramíneas. Essas plantas daninhas possuem alto nível de agressividade para a cultura, e uma grande porcentagem de ocupação desse tipo de planta na região pode indicar alto risco de infestação, devido à sua alta proliferação.

\section{RESULTADOS E DISCUSSÃO}

O mapa de densidade de sementes é estimado a partir de dados simulados para a sexta geração usando a equação 1 e o mapa de cobertura foliar a partir de dados simulados para a quinta geração das plantas daninhas, usando a equação 2. Para tornar o modelo (eq. 1) mais representativo de uma população real, o parâmetro $g$ é gerado aleatoriamente pelo método de Monte Carlo a partir da distribuição normal em torno do ponto $g=0,1 \mathrm{com}$ um desvio de média 0 e variância 0,04 , ou seja, $g \sim N(0,1,0,04)$. Foram gerados 100 valores para a cobertura foliar e densidade de sementes.

Os diferentes modelos de semivariograma, como o gaussiano, esférico e exponencial, foram testados, e este último foi escolhido, por ter apresentado o menor índice de ajuste (Iwashita \& Landim, 2003). A Figura 2 ilustra a curva do semivariograma para a sexta geração ajustada aos pontos que representam os pares de valores de densidade de mesma distância. A partir deste semivariograma, pode-se notar a dependência espacial entre as observações até o alcance de $20 \mathrm{~m}$. Os mapas interpolados por krigagem para uma área de $10.000 \mathrm{~m}^{2}$ são exibidos na Figura 3.

Os mapas da Figura 3 são tratados como imagens. Objetos identificados nestes mapas fornecem atributos para a infestação. Para isto, os mapas foram divididos em 33 pixels de 3x3 metros por eixo. Assim, obtém-se uma matriz A de tamanho 33x33, cujos elementos são os valores das intensidades em cada pixel, tanto para a densidade de semente quanto para a cobertura foliar.

Os objetos associados aos mapas são obtidos a partir da matriz A da seguinte forma. Primeiramente, estipula-se 3 faixas de intensidades da matriz A, em que a primeira é representada por preto, a segunda por cinza e a terceira por branco. Os limitantes destas faixas são definidos a partir do mínimo e do máximo valor de intensidade encontrado em A. Desta forma, gera-se uma matriz I, de tamanho 33x33, cujos elementos são 0, 1 ou 2 , formando diferentes objetos. Estes, mesmo podendo apresentar a mesma intensidade, podem constituir objetos diferentes da imagem.

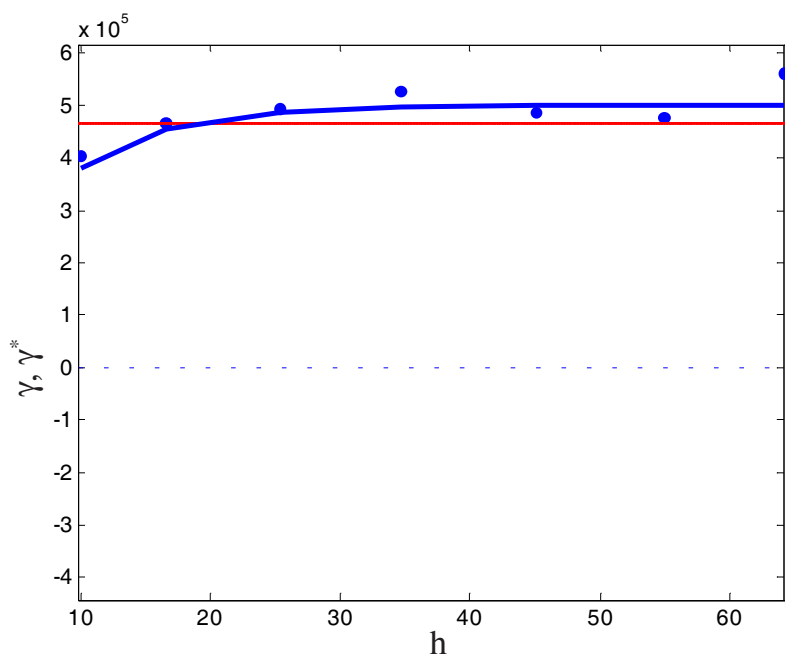

Figura 2 - Ajuste do semivariograma teórico para a sexta geração de sementes. 
Em seguida, para identificar objetos conectados, adota-se um modelo de vizinhança 4-conectado (Gonzalez e Woods, 2000) para a agregação de cada pixel a um objeto. Este modelo é implementado primeiramente convertendo a matriz I em uma matriz binária. Então, os 4 vizinhos mais próximos de um pixel são analisados e os que possuírem o mesmo valor, 0 ou 1, são agregados. Finalmente, os objetos conectados são rotulados e estes rótulos são organizados em uma matriz J, também de dimensão 33x33, representada na escala de cinza. Os pixels rotulados como 0 constituem um objeto conectado, os rotulados como 1 constituem um segundo objeto conectado, e assim por diante.

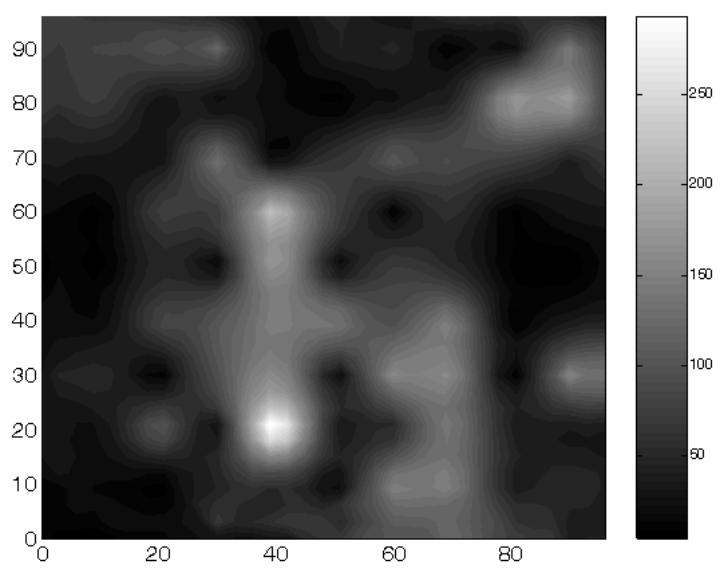

(a)
A combinação de todos os atributos definidos na seção anterior em suas diversas condições fornece uma base de regras fuzzy. A saída do sistema de classificação, $c_{i}$, $i=1, \ldots, C$, é o risco de infestação por região, descrito por variáveis lingüísticas como alto, médio e baixo. Por motivo de simplificação e redução do tempo de execução, adotam-se funções de pertinência triangulares para as entradas e a saída. O método do mínimo produto é usado na composição de regras de inferência, e o método do máximo, para agregar as regras (Pedrycz \& Gomide, 1998). A Tabela 1 exibe a base de regras fuzzy para a determinação do risco de infestação de cada região da cultura. Por exemplo, a regra

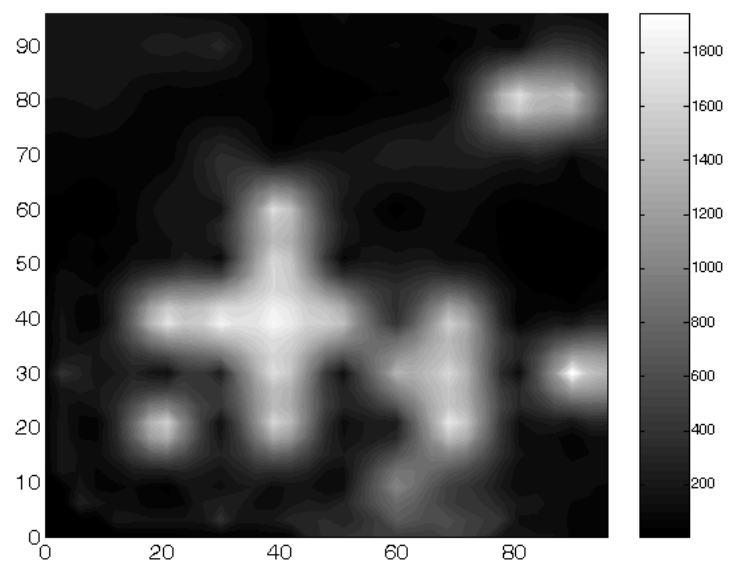

(b)

Figura 3 - (a) mapa de cobertura foliar de plantas daninhas na quinta geração, (b) mapa da densidade de sementes de plantas daninhas na sexta geração para $s=248$ ou $s=500, a=4,0 \times 10^{-3}, b=2,77, c=0,9, X_{0}=1, g \sim N(0,1,0,04)$ e um limiar máximo de 4.000 sementes $\mathrm{m}^{-2}$ para a produção de sementes por planta na próxima geração.

Tabela 1 - Base de regras fuzzy para o risco de infestação por plantas daninhas

\begin{tabular}{|c|c|c|c|c|c|c|c|c|c|}
\hline \multicolumn{10}{|c|}{ Densidade de sementes/Extensão do objeto } \\
\hline Cobertura/Ocupação & $\begin{array}{c}\text { baixa/ } \\
\text { pequena }\end{array}$ & $\begin{array}{l}\text { baixa/ } \\
\text { regular }\end{array}$ & $\begin{array}{l}\text { baixa/ } \\
\text { grande }\end{array}$ & $\begin{array}{c}\text { média/ } \\
\text { pequena }\end{array}$ & $\begin{array}{l}\text { média/ } \\
\text { regular }\end{array}$ & $\begin{array}{l}\text { média/ } \\
\text { grande }\end{array}$ & $\begin{array}{c}\text { alta/ } \\
\text { pequena }\end{array}$ & alta/ regular & alta/grande \\
\hline rala/baixa & $c_{11}$ & $\mathrm{c}_{16}$ & $c_{110}$ & $\mathrm{c}_{113}$ & $\mathrm{c}_{116}$ & $c_{117}$ & $c_{118}$ & $c_{119}$ & $c_{120}$ \\
\hline rala/média & $\mathrm{c}_{12}$ & $c_{17}$ & $\mathrm{c}_{28}$ & $c_{211}$ & $c_{215}$ & $c_{219}$ & $\mathrm{c}_{23}$ & $c_{227}$ & $c_{231}$ \\
\hline rala/alta & $c_{13}$ & $c_{24}$ & $\mathrm{c}_{29}$ & $c_{212}$ & $\mathrm{c}_{38}$ & $c_{312}$ & $c_{316}$ & $c_{320}$ & $c_{324}$ \\
\hline moderada/baixa & $\mathrm{c}_{14}$ & $\mathrm{c}_{18}$ & $c_{111}$ & $c_{114}$ & $c_{216}$ & $c_{220}$ & $c_{224}$ & $c_{228}$ & $c_{232}$ \\
\hline moderada/média & $c_{21}$ & $c_{25}$ & $c_{210}$ & $c_{213}$ & $c_{217}$ & $c_{221}$ & $c_{225}$ & $c_{229}$ & $c_{233}$ \\
\hline moderada/alta & $\mathrm{c}_{22}$ & $\mathrm{c}_{26}$ & $\mathrm{c}_{33}$ & $\mathrm{c}_{36}$ & $\mathrm{c}_{39}$ & $c_{313}$ & $c_{317}$ & $c_{321}$ & $c_{325}$ \\
\hline densa/baixa & $c_{15}$ & $c_{19}$ & $c_{112}$ & $c_{115}$ & $c_{218}$ & $c_{222}$ & $c_{226}$ & $c_{230}$ & $\mathrm{c}_{234}$ \\
\hline densa/média & $c_{23}$ & $\mathrm{C}_{27}$ & $\mathrm{c}_{34}$ & $c_{214}$ & $c_{310}$ & $\begin{array}{l}c_{314} \\
3\end{array}$ & $c_{318}$ & $c_{322}$ & $c_{326}$ \\
\hline densa/alta & $\mathrm{c}_{31}$ & $\mathrm{c}_{32}$ & $\mathrm{c}_{35}$ & $\mathrm{c}_{37}$ & $c_{311}$ & $c_{315}$ & $c_{319}$ & $c_{323}$ & $c_{327}$ \\
\hline
\end{tabular}

O primeiro subscrito denota a classe de infestação: 1 - baixa infestação, 2 - média infestação e 3 - alta infestação; o segundo denota quantas vezes a classe do primeiro subscrito está sendo citada. 
destacada na Tabela 1 é entendida como segue:

SE a densidade de sementes na região é baixa $\mathrm{E}$ a extensão dos objetos na região é pequena $\mathrm{E}$ a cobertura foliar na região é rala $\mathrm{E}$ a agressividade é baixa ENTÃO classe de baixo risco de infestação.

Para exemplificar o cálculo dos atributos, consideram-se regiões da imagem de tamanho $7 \times 7$ pixels, cuja medida em metros obedece à dependência espacial determinada pelas análises variográficas. Portanto, a cultura é dividida em 25 regiões e os atributos da infestação são calculados para estas regiões.

\section{Cálculo dos Atributos}

Os atributos da infestação são entradas do sistema de classificação fuzzy, e a saída deste sistema fornece o risco de infestação por plantas daninhas de cada uma das regiões consideradas da cultura. O procedimento para obtenção dos valores dos $X_{i k}, i=1,2,3,4$ atributos em cada região é descrito a seguir.

$X_{i k}$ : O valor médio de densidade de sementes de cada região, associado à produção de sementes, é obtido a partir da média ponderada entre as intensidades dos objetos conectados e o n[umero de pixels ocupados por estes, pois cada região pode conter vários objetos que representam um mesmo valor de densidade. $\mathrm{O}$ atributo densidade média de sementes para as 25 regiões da cultura, para a sexta geração, é organizado como na matriz $X_{1}$, apresentada a seguir:

$$
X_{1}=\left[\begin{array}{lllll}
0,1667 & 0,1667 & 0,1667 & 0,1762 & 0,2133 \\
0,1667 & 0,1735 & 0,1735 & 0,2041 & 0,2381 \\
0,1769 & 0,3061 & 0,2687 & 0,1871 & 0,1667 \\
0,1939 & 0,3469 & 0,3129 & 0,3401 & 0,2667 \\
0,1769 & 0,1905 & 0,2007 & 0,2245 & 0,1667
\end{array}\right]
$$

Na Figura 4a pode-se observar o mapa dos objetos que constituem a imagem da Figura 3b. A Figura 4b exibe as correspondentes 25 regiões, cada uma representando um valor médio de densidade de sementes, de acordo com a matriz $X_{1}$.

$X_{2 k}$ : A cobertura foliar por região é obtida da mesma maneira que o atributo $X_{1 k}$. A matriz de cobertura foliar média, denotada $X_{2}$, é a seguinte:

$$
X_{2}=\left[\begin{array}{lllll}
0,1667 & 0,1714 & 0,1667 & 0,1762 & 0,2333 \\
0,1667 & 0,1973 & 0,1769 & 0,1939 & 0,2190 \\
0,1667 & 0,2279 & 0,2279 & 0,1769 & 0,1667 \\
0,1667 & 0,2517 & 0,2687 & 0,2585 & 0,2095 \\
0,1667 & 0,1837 & 0,2041 & 0,2517 & 0,1667
\end{array}\right]
$$

Do ponto de vista da distribuição das plantas emergentes, o pior caso seria uma só reboleira preenchendo todos os pixels da

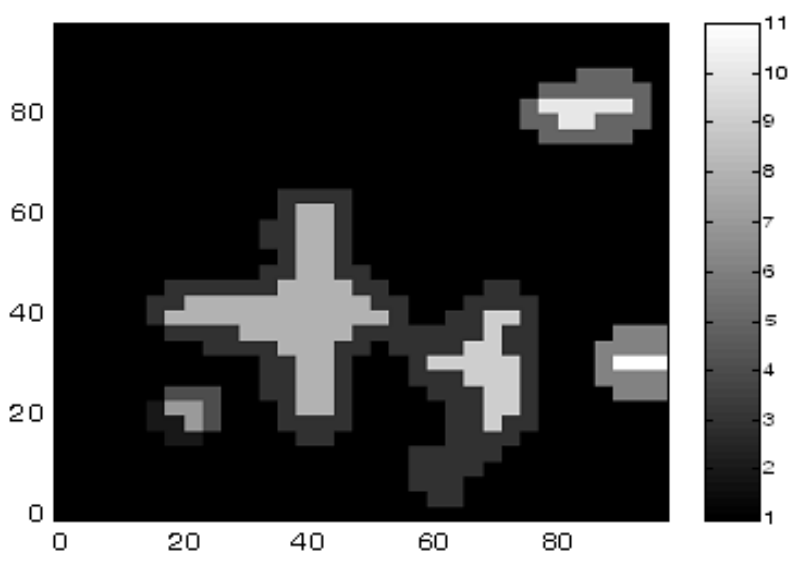

(a)

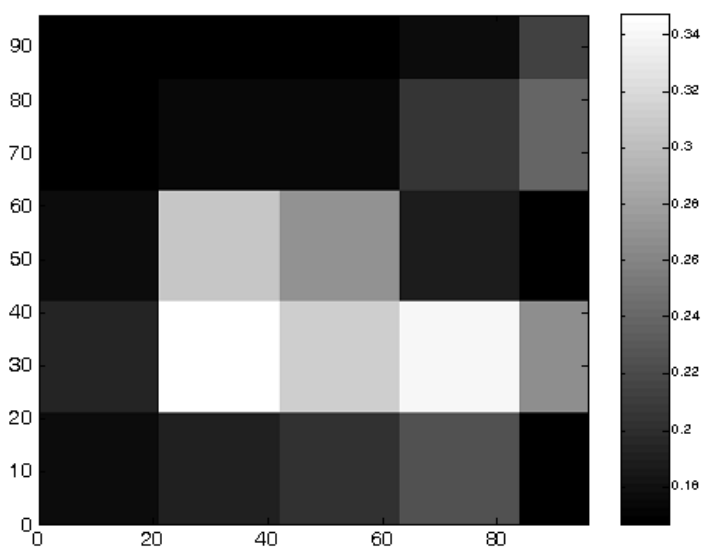

(b)

Figura 4 - a) objetos conectados identificados no mapa de densidade de sementes; e (b) regiões de valor médio ponderado de densidade, correspondentes à $X_{I}$. 
região, representando 100\% de ocupação. Na Figura 5a pode ser observado o mapa dos objetos que constituem a imagem da Figura 3a. A Figura $5 \mathrm{~b}$ exibe as correspondentes 25 regiões, cada uma representando um valor médio de cobertura foliar, de acordo com a matriz $X_{2}$.

$X_{3 k}$ : A extensão média dos agrupamentos de densidade de sementes em cada região é obtida a partir do número de pixels de cada objeto da Figura 4a nesta região. Assim, da mesma forma que nos casos anteriores, obtémse a matriz de extensão média dos objetos, denotada $X_{3}$, como segue:

$$
X_{3}=\left[\begin{array}{lllll}
0,1667 & 0,1667 & 0,1667 & 0,1762 & 0,2133 \\
0,1667 & 0,1735 & 0,1735 & 0,2041 & 0,2381 \\
0,1769 & 0,3061 & 0,2687 & 0,1871 & 0,1667 \\
0,1650 & 0,1973 & 0,2517 & 0,3401 & 0,2667 \\
0,1769 & 0,1667 & 0,2007 & 0,2245 & 0,1667
\end{array}\right]
$$

Se um determinado valor de densidade de sementes estiver ocupando uma grande parte da região, isto é, grande número de pixels, então este objeto, constituído por estes pixels, terá maior influência no cálculo da extensão dos objetos em cada região, através da média ponderada. A Figura 6 exibe as 25 regiões referentes à extensão média dos objetos que representam a ocupação da densidade de sementes em cada região da cultura, de acordo

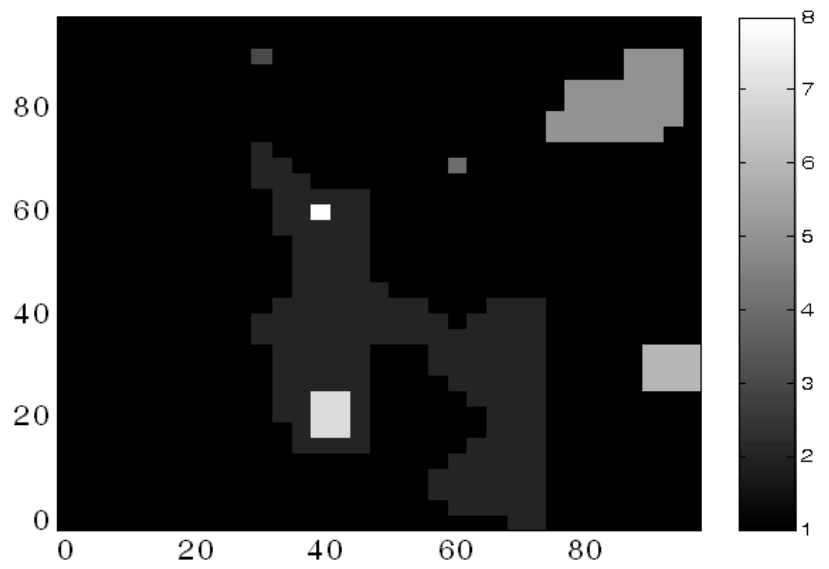

(a)

Figura 5 - (a) objetos conectados identificados no mapa de cob correspondentes à $X_{2}$.

Planta Daninha, Viçosa-MG, v. 24, n. 2, p. 229-238, 2006 com os valores das médias de extensão dadas pela matriz $X_{3}$.

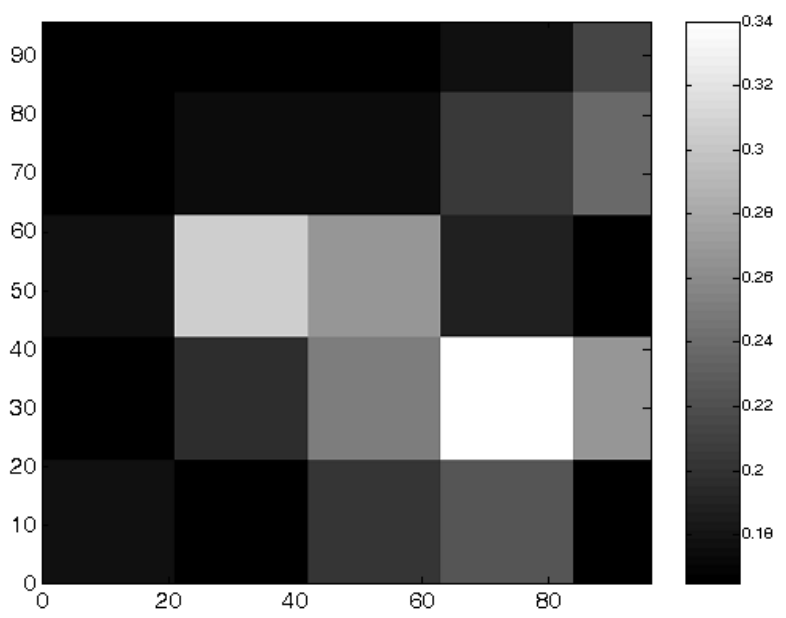

Figura 6 - Regiões de valor médio de extensão dos objetos.

$X_{4 k}$ : A agressividade das plantas daninhas presentes em cada região é calculada considerando a porcentagem de ocupação de espécies com alta capacidade de produção de sementes. Essa porcentagem é calculada com o modelo (1) contando o número de amostras obtidas com $\mathrm{s}=500$. A agressividade das plantas daninhas em cada região é exibida na matriz denotada $X_{4}$.

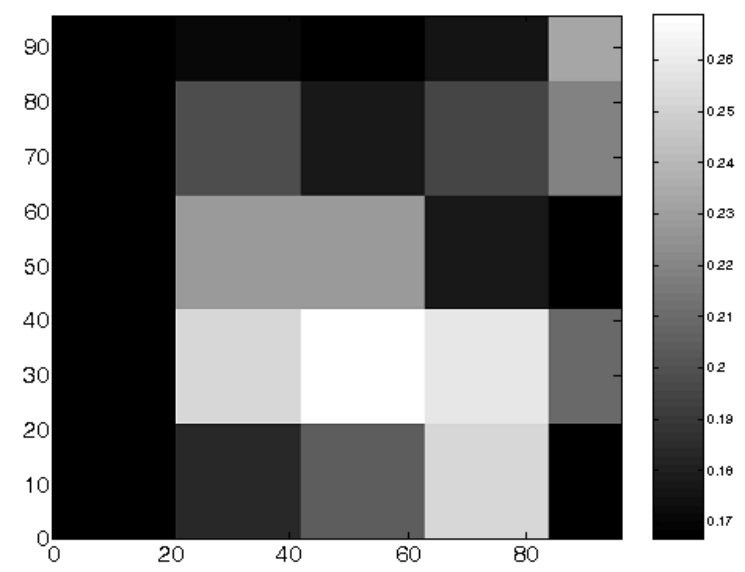

(b)

ra foliar; e (b) regiões de valor médio ponderado de cobertura, 


$$
X_{4}=\left[\begin{array}{lllll}
0,0000 & 0,0000 & 0,0000 & 0,0000 & 0,0000 \\
0,0000 & 0,0000 & 0,0000 & 0,2500 & 0,2500 \\
0,0000 & 0,5000 & 0,0000 & 0,0000 & 0,0000 \\
0,2500 & 0,7500 & 0,5000 & 0,5000 & 0,5000 \\
0,2500 & 0,2500 & 0,2500 & 0,2500 & 0,0000
\end{array}\right]
$$

A classificação do risco de infestação descrita pelos atributos definidos na seção anterior foi realizada para dois casos: o primeiro, dividindo a cultura em 25 regiões, descritas anteriormente; e o segundo, considerando toda a cultura como uma só região, obtendo apenas um risco de infestação. Para implementar o sistema de classificação fuzzy, foi utilizado o editor de sistemas de inferência fuzzy do MATLAB. As funções de pertinência adotadas para as variáveis de entrada e de saída são apresentadas na Figura 7. A função de pertinência adotada para a saída inclui uma faixa de valores negativos para a parte inferior da função "baixa" e uma faixa de valores acima de $100 \%$ para a parte superior da função "alta" somente para efeito de cálculo, a fim de garantir que a menor infestação seja nula e a maior seja $100 \%$.

Aplicando o modelo de classificação fuzzy regionalmente, segundo os valores dos atributos calculados, obtêm-se 25 valores diferentes de risco de infestação da cultura, um para cada região considerada. Esses valores constituem a matriz denotada Risco:

$$
\text { Risco }=\left[\begin{array}{lllll}
0,3500 & 0,3550 & 0,3560 & 0,3700 & 0,4000 \\
0,3800 & 0,3900 & 0,3800 & 0,4200 & 0,4200 \\
0,3700 & 0,5400 & 0,4500 & 0,4100 & 0,4000 \\
0,4000 & 0,6000 & 0,5500 & 0,5800 & 0,5220 \\
0,4000 & 0,4200 & 0,4000 & 0,4500 & 0,4100
\end{array}\right]
$$

em que cada elemento corresponde ao risco de infestação da respectiva região. Dessa forma, tem-se que a primeira região da plantação apresenta risco de infestação de $35 \%$, enquanto a segunda região apresenta $35,5 \%$, e assim por diante.

Por outro lado, considerando toda a cultura homogeneamente, como uma única região, obtém-se um único risco de infestação por plantas daninhas em toda a área considerada. Os valores dos atributos calculados para este caso são os seguintes: $X_{1}=0,2175$, $X_{2}=0,1996, X_{3}=0,1393$ e $X_{4}=0,1800$. A combinação desses atributos no sistema de classificação fuzzy resulta num risco de infestação de 37\% para toda a cultura, sugerindo um tratamento médio homogêneo.

Os resultados de simulação mostram que, se o risco de $37 \%$ de infestação for assumido para a tomada de decisão quanto à aplicação de herbicida, o risco de infestação de algumas regiões, como as correspondentes às posições $(3,2),(4,2),(4,3)$ e $(4,4)$ da matriz Risco, que representam respectivamente 54, 60, 55 e 58\% de risco de infestação, seria subestimado. Além disso, se o maior risco de infestação, que é $60 \%$, for considerado e apenas uma taxa de herbicida correspondente a este for aplicada, outras regiões receberiam mais defensivos que o necessário para o controle da infestação. Portanto, a aplicação de recomendações médias de herbicidas para toda a cultura pode resultar em uma super ou subaplicação em algumas regiões. Dessa forma, o mapa do risco de infestação por regiões considera a variabilidade espacial das plantas daninhas, aumentando a eficiência do insumo, reduzindo custos e o impacto ambiental, tornando o ambiente agrícola mais bem controlado.
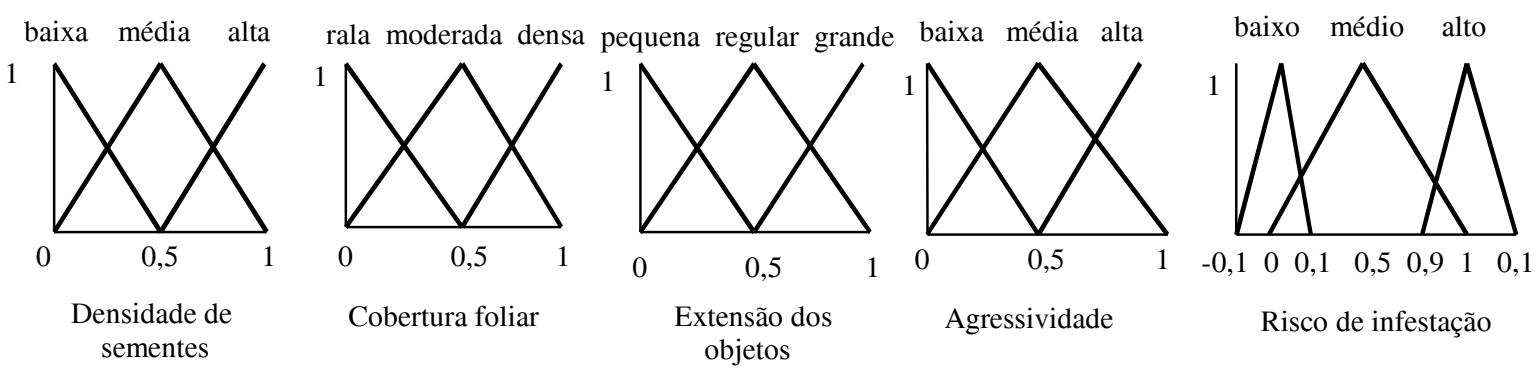

Figura 7 - Funções de pertinência das entradas e da saída do sistema de classificação fuzzy. 


\section{AGRADECIMENTOS}

À CAPES, pelas bolsas de estudo concedidas aos dois primeiros autores.

\section{LITERATURA CITADA}

AITKENHEAD, M. J. et al. Weed and crop discrimination using image analysis and artificial intelligence methods. Comp. Electr. Agric., v. 39, n. 3, p. 157-171, 2003.

BAIO, F. H. R.; BALASTREIRE, L. A. Aplicação localizada de defensivos baseada na variabilidade espacial das plantas daninhas. 2001. 13 f. Dissertação (Mestrado em Agronomia). Escola Superior de Agricultura "Luiz de Queiroz", Piracicaba, 2001.

CARVALHO, J. R. P.; VIEIRA, S. R. Avaliação e comparação de estimadores de krigagem para variáveis agronômicas - uma proposta. Campinas: EMBRAPA Informática Agropecuária, 2001. p. 1-20. (Documento, 3)

CHIANG, J.; HSU, J. Fuzzy classification trees for data analysis. Fuzzy Sets Syst., v. 130, n. 1, p. 87-99, 2002.

CRUVINEL, P. E. et al. Sistema computacional de baixo custo para reconhecimento da variabilidade de plantas daninhas baseado em imageamento estereoscópico. In: CONGRESSO BRASILEIRO DE AGRICULTURA DE PRECISÃO - CONBAP, 2004, Piracicaba. Anais... Piracicaba: 2004. p. 1-14.

GONZALEZ-ANDUJAR, J. L. High control measures cannot produce extinction in weed populations. Ecol. Modelling, v. 91, n. 1-3 p. 293-294, 1996.
GONZALEZ, R. C.; WOODS R. E. Processamento de imagens digitais. São Paulo: Edgard Blücher, 2000. 509 p.

ISAAKS, E. H.; SRIVASTAVA, R. M Applied Geostatistics. Oxford: University Press, 1989. 580 p.

IWASHITA, F; LANDIM, P. B. GEOMATLAB: Análise geoestatística usando MATLAB, DGA, IGCE. Rio Claro: Universidade Estadual de São Paulo, 2003. 17 p. (Texto didático, 12)

KAVDIR, I.; GUYER, D. E. Apple grading using fuzzy logic. Turk. J. Agric. For., v. 27, n. 6, p. 375-382, 2003.

PEDRYCZ, W.; GOMIDE, F. An introduction to fuzzy sets. Cambridge, MA: MIT Press, 1998. 480 p.

PEIXOTO M. S.; BARROS, L. C.; BASSANEZI, R. C. Um modelo Fuzzy Presa-Predador em citros: pulgões e joaninhas. Biomatemática, v. 14, p. 29-38, 2004.

RIZZARDI, M.; FLECK, N. G. Métodos de quantificação da cobertura foliar da infestação de plantas daninhas e da cultura da soja. Ci. Rural, v. 34, n. 1, p.13-18, 2004.

SHIRATSUCHI, L. S.; CHRISTOFFOLETI, P. J. Mapeamento da variabilidade espacial das plantas daninhas com a utilização de ferramentas da agricultura de precisão. 2001. 96 f. Dissertação (Mestrado em Agronomia) - Escola Superior de Agricultura "Luiz de Queiroz", Piracicaba, 2001.

VIEIRA, S. R.; LOMBARDI, F.; BURROWS, I. T. Mapeamento da chuva máxima provável para o Estado de São Paulo. R. Bras. Ci. Solo, v. 15, n. 1, p. 93-98, 1981.

YANG, C. C. et al. Development of a herbicide application map using artificial neural networks and fuzzy logic. Agric. Syst., v. 76, n. 2, p. 561-574, 2003. 